\title{
Contrast-based digital tracking versus human observers in studies of animal locomotion
}

\author{
MICHAEL J. RENNER, PETER J. PIERRE, and PATRICK J. SCHILCHER \\ University of Wisconsin, Oshkosh, Wisconsin
}

\begin{abstract}
The availability of relatively inexpensive digital contrast detection creates the possibility of automated, general-purpose motion tracking, equally applicable to concurrent remote video observation and analysis of videotape recordings. In this study, a digital method of scoring locomotor activity in a laboratory arena was compared with scoring by human observers. The digital tracking device (HVS VP-112) utilized foreground/background contrast to determine subject location from a video image; human observers scored from videotape. In addition, relative floor brightness (red lighting was held constant) was manipulated to compare the efficiency of the digital tracking system's ability to detect a moving subject under different contrast conditions. The arena had a reversible floor, with opposite sides dark gray and white. Six subjects (male LongEvans Rattus norvegicus, 4 months old) were given opportunity to enter the arena for the first 2 days on the white floor; the floor was reversed for the 3rd day. Six matched subjects followed an opposite schedule. Recorded measures of zone changes, made by digital tracking and by humans, were highly correlated $(r=.951, p<.001)$ and not significantly different. Activity level, as measured by number of entries, latency to enter, and total time in the arena, was significantly higher in subjects for which the dark floor was showing on Day 1 . Although these scoring methods produced comparable results, requisite constraints on experimental procedures may limit the usefulness of this type of tracking device.
\end{abstract}

There are several means with which an animal's activity in an open field can be measured. The oldest is the manual scoring method, in which an observer records the subject's location and activities. Manual scoring requires either direct or remote observation. Unlike remote observation, the observer's presence during direct observation can bias the subject's behavior; remote observation removes any possible influence of the experimenter's presence.

Automated methods of scoring include systems of touch plates mounted below the arena floor (Pfister, Mudge, \& Harcombe, 1978; Porter, Hudy, \& Furber, 1979); detection of relocation when the animal breaks a fixed arrangement of infrared beams in the open field; and, recently, computer-interfaced analysis of a video image (Jouen \& Lepecq, 1989; Livesey \& Leppard, 1981; Lubinski, Dickson, \& Cairns, 1977). Both touch plates and infrared beams are tied to specific apparatuses. Videobased analysis is appealing because of its potential for flexible application: One system can be used in multiple situations.

In this study, a video-based automated method of scoring animal locomotion was compared with human scoring of the same videotape records. The particular system used (HVS Image Systems model VP-112) compares fore-

Michael J. Renner and Peter J. Pierre are now at Memphis State University. Address correspondence concerning this article to Michael J. Renner, Psychology Department, Memphis State University, Memphis, TN 38152. ground/background contrast (either light on dark or dark on light) and determines the subject's location from the video image by locating the first pixel group in the scanning pattern that meets the preset criterion for contrast.

Since the image analyzer locates the subject by detecting contrast, and contrast can be affected by the amount of ambient light in the arena, floor color was also manipulated; floors of different brightness (i.e., reflectance) would provide different ambient light levels at the same illumination. Previous research has indicated that a darker floor shade increases both the behavioral activity and the physiological arousal of the rat (Hall, 1934; Hirsjärvi \& Junnila, 1986; Hlinak \& Rozmarova, 1986). While studying the effect of illumination on the activity level of rats, Valle (1970) found activity (measured by mean number of squares-zones-entered and by the mean number of rears) was significantly greater under darker conditions.

The ready availability of microcomputers and "off-theshelf' image-analysis equipment of moderate cost make the use of contrast-based location tracking a realistic alternative to hand scoring. This experiment was undertaken to compare the relative usefulness of contrast-based digital analysis of motion with human scoring methods.

\section{METHOD}

\section{Subjects}

Twelve male, 4-month old, Long-Evans hooded rats (Rattus norvegicus) bred in-house from stock obtained from Harlan Sprague-Dawley (Indianapolis, IN), were used as subjects. The rats had no prior experience in any arena. They were fed a standard laboratory diet, main- 
tained on a 12:12-h light:dark cycle. Two days before data collection, the rats were individually housed in polycarbonate tub cages $(19 \times 36$ $\times 24 \mathrm{~cm})$, which were equipped with wooden cubes $(10 \mathrm{~cm} / \mathrm{side})$ to facilitate their entry into the arena.

\section{Materials}

Experimental sessions were recorded in an open-field arena $(120 \mathrm{~cm}$ square) with clear plexiglass sides $(60 \mathrm{~cm}$ in height). In order to provide an objective basis for manual scoring, black paint lines $1 \mathrm{~cm}$ wide were painted on the arena floor, dividing it into 9 equal zones arranged as a $3 \times 3$ grid. The entrance from the startbox was a $10-\mathrm{cm}$ hole in the floor of the middle zone of the arena. A reversible arena insert (with opposing gray and white sides) and color-matched startbox blind, constructed from $1 / 4$-in. plywood, were used to manipulate arena floor brightness.

Sessions were videotaped with a single low-light video camera (Panasonic WV-1550) mounted directly overhead and equipped with a 16mm lens. With the exception of the video camera, all data-gathering equipment was placed in an adjacent room. The video signal was fed through a time/date generator (Panasonic WJ-810) to a VHS format VCR (Sharp XA-110). Data tapes were viewed on a videotape system that included a Panasonic video recorder and monitor (Models NV-8950 and BT-S1900N). The motion-analysis equipment consisted of an MS- DOS computer system (Zenith ZF-148), a VCR (Sharp XA-110), a video monitor (Panasonic TR-931), and an image analyzer (HVS VP-112).

Data were collected under red lighting. The arena was illuminated by four $25-\mathrm{W}$ red incandescent bulbs (two metal reflective cones and two plastic diffuser/reflector cones) in both floor conditions. Pilot testing and previous studies have shown this form of lighting to be functionally invisible to rats. The bulbs were directed toward opposing corners to control for possible "hot spots" that could be detected by the HVS and be read as contrast. To provide the animals with a low level of visible illumination, a 25-W white incandescent light was placed in a metal reflector, which was directed at a wall from a distance of $4 \mathrm{~cm}$ approximately $2.5 \mathrm{~m}$ above, and $2 \mathrm{~m}$ to one side of, the arena.

\section{Software Description}

The manual data-collection process was aided through the use of behavioral observation software, written by Renner (1989) in BASIC and running on an MS-DOS microcomputer. This program empties the keyboard buffer and uses the operating system clock to record once per second what key (if any) is being pressed.

Boundaries of the tracking field could be established with controls on the HVS system; the TRACKER software program (Renner \& Laurion, 1989) allowed the division of this defined field into any number of divisions along both $x$ and $y$ axes. In this experiment, nine square zones were used (in a $3 \times 3$ grid, each zone $40 \mathrm{~cm}$ per side), isomorphic with the zones used in manual scoring.

\section{Design and Procedure}

To begin each session, the rat's cage was placed on an adjustable shelf suspended below the arena floor, so that the open top of the cage was flush against the underside of the floor. The rats could gain access to the arena through the $10-\mathrm{cm}$ hole in the center of the arena floor. Video recording equipment was started after each animal had been given access to the arena and the experimenter had left the experimental room and closed the door. The rats had unrestricted access to the arena and the home cage for the entire session, and they could choose to remain out of the arena by staying in the home cage.

Recording sessions, $10 \mathrm{~min}$ long, spanned three consecutive nights. On Days 1 and 2, half the rats were exposed to the light arena-floor shade condition and half were exposed to the dark floor. On Day 3, the floor was reversed for each group.

\section{Scoring}

Manual. For manual scoring, practiced observers mapped the rat's movements onto the numerical keypad of the computer; these numbers corresponded directly to numbers assigned to the nine zones of the arena. A zone change was defined as the rat's nose and forepaws crossing from one zone to another.

Contrast-based tracking. The HVS measured foreground/background contrast. In this study, the hood of the rat contrasted with the light floor and the white body parts contrasted with the dark floor. The HVS read $x$ and $y$ coordinates, starting from the upper right corner of the established field, and continuing until it registered a point of sufficient contrast, which was the first point of contrast that surpassed the HVS's preset lower limen. The rectangular size of the scanning field was established by setting $x$ and $y$ limiting points on the HVS. Objects could be located with the video image with a precision limited by the image analyzer's $256 \times 230$ pixel resolution. For both scoring methods, the index of locomotor activity was defined in a manner similar to that originally defined by Hall and Ballachey (1932) as the number of zone changes.

\section{RESULTS}

Manual and contrast-based methods of scoring produced counts of the number of zone changes that were not significantly different $[t(35)=-1.333, p=.1882]$. Scores from the two scoring methods were highly correlated $[r(34)=.95, p<.001]$. A mixed-design $2 \times 3$ ANOVA (floor color [between subjects] $\times$ days [within subjects]) revealed that the rats entered the arena more quickly if the dark floor was used on Day $1[F(1,10)=11.93$, $p<.01$; see Figure 1]. The rats in the dark-floor-first group showed a significantly greater number of arena en-

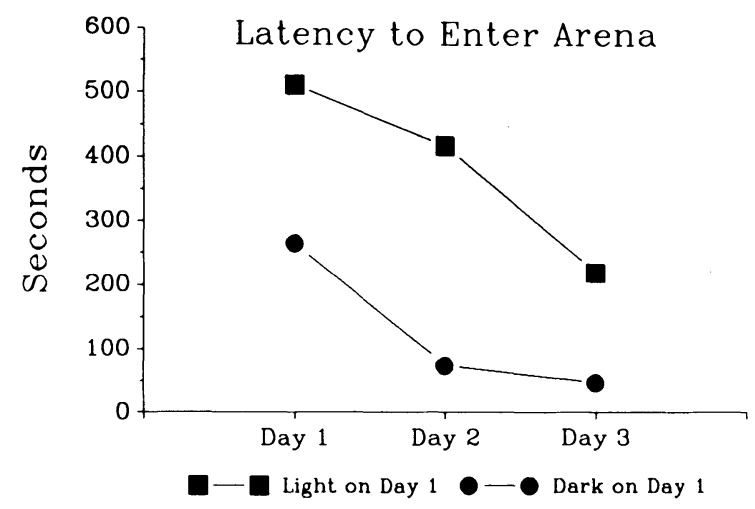

Figure 1. Latency to enter the arena. Rats entered the arena more quickly on the dark floor $[F(1,10)=11.93, p<.01]$. The effect of days was also significant $[F(2,33)=10.85, p<.001]$. The interaction was nonsignificant.

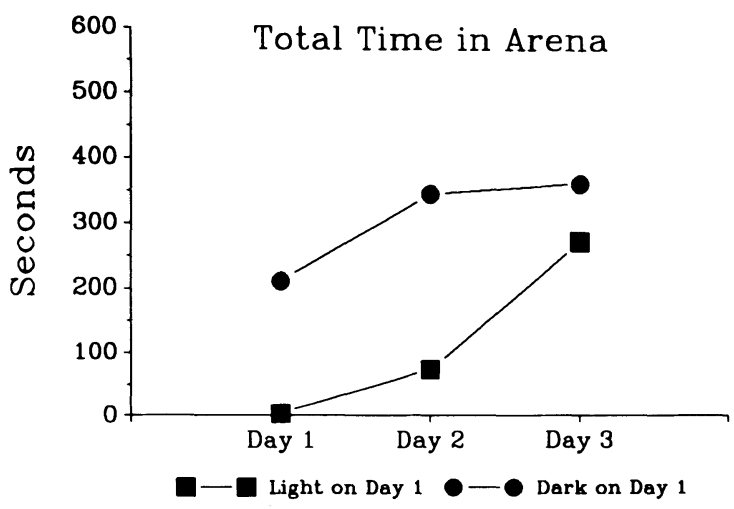

Figure 2. Total time in the arena. Rats spent more time in the arena on the dark floor $[F(1,10)=13.78, p<.01]$, and increased the time spent in the arena over days $[F(2,33)=10.60, p<.001]$. The interaction was nonsignificant. 
tries $[F(1,10)=16.23, p<.005]$. The rats in the darkfloor-first group also spent more total time in the arena $[F(1,10)=13.77, p<.005$; see Figure 2].

\section{DISCUSSION}

The near-perfect correlation between the manual and contrast-based (HVS image analyzer) modes of location measurement established that the two methods are equivalent for obtaining an index of animal locomotor activity. In addition, rats' preference for a darker environment was shown by shorter emergence latency and longer time spent in the arena on a dark floor. These results are consistent with conclusions found in previous research on illumination and activity (Hlinak \& Rozmarova, 1986; Valle, 1970).

The equivalence between the two modes of measurement is promising because of the system's potential for scoring locomotion from previously videotaped sessions. Hypothetically, the only constraints on the size of the tracking field would be those imposed by the limits on what can be successfully recorded on videotape. Such a system could be used in a variety of experimental designs, regardless of the anatomical size of the subject, providing the subject could be captured in a video image. In addition, the equipment necessary for video image analysis is becoming increasingly affordable-and therefore available-due to recent advances in integrated circuit technology.

There are potentially serious difficulties, however, that must be recognized when one plans studies that might use a contrast-based tracking system such as the HVS VP-112. Since this type of system relies on contrast between the subject and the surrounding environment, successful tracking depends on the degree to which contrast can be controlled. This difficulty becomes clear in the event that the subject effects a change in environment-for example, a change in surface reflection due to urination, or a change of an object's position due to the subject's interaction with that object. It is necessary to consider the contrast between the arena floor and the subject, as well as the shades of any objects introduced into the experimental situation. Our study raised a technical issue of subject coloration: with the use of hooded rats, different body parts are tracked in the dark-on-light and light-on-dark situations. Objects in the arena must be flat black or white and shadows in the experimental environment must be carefully controlled, not only for the effect of contrast, but also because of the rat's preference for darker areas. This tends to encourage overillumination of the arena. The accumulated total of situational constraints in the methodology presented, required by the use of contrast-based tracking, might alter a particular experimental design to such a degree that the data collected are no longer equivalent to data collected by other means.
The possible utility of recording locomotor activity via contrast-based video image analysis should not be completely overshadowed by the controls that are necessary in order for one to utilize such a system. At present, however, the technology is not sufficiently advanced for it to be successfully incorporated into many existing methods of observational data collection.

\section{REFERENCES}

Hall, C. S. (1934). Emotional behavior in the rat. Journal of Comparative Psychology, 18, 385-403.

HAll, C. S., \& BallacheY, E. L. (1932). A study of the rat's behavior in a field: A contribution to method in comparative psychology. University of California Publications in Psychology, 6, 1-12.

HiRSJÄRVI, P. A., \& JUNNILA, M. A. (1986). Effects of light and noise test stimuli on the open-field behavior of Wistar rats. Scandinavian Journal of Psychology, 27, 311-319.

Huinak, Z., \& Rozmarova, E. (1986). The locomotor-exploratory behavior of laboratory male rats tested under the "red" and "white" light conditions. Activitas Nervosa Superior, 28, 202-203.

Jouen, F., \& LEPECQ, J.-C. (1989). Optical and electronic systems for spatial and temporal analysis of video images. Behavior Research Methods, Instruments, \& Computers, 21, 2-10.

LiveseY, P. J., \& LePPARD, K. (1981). A TV monitored system for recording open-field activity in the rat. Behavior Research Methods \& Instrumentation, 13, 331-333.

LUbinski, K. S., Dickson, K. L., \& CaIRns, J. (1977, December). Microprocessor-based interface converts video signals for object tracking. Computer Design, pp. 81-87.

Pfister, H. P., Mudge, R. R., \& Harcombe, A. O. (1978). A multipurpose activity platform utilized in the open-field setting. Behavior Research Methods \& Instrumentation, 10, 21-22.

Porter, J. J., Hudy, J. J., \& FURBer, A. M. (1979). A pressure-actuated open-field apparatus for rodents. Behavior Research Methods \& Instrumentation, 11, 59-60.

RENNER, M. J. (1989). Location and general activities program [Unpublished computer program].

ReNNER, M. J., \& LAURION, L. (1989). Tracker: Computer program developed to track animal locomotion. Unpublished computer program.

VALLE, F. P. (1970). Effects of strain, sex, and illumination on openfield behavior of rats. American Journal of Psychology, 83, 103-111.

(Manuscript received June 23, 1989.) 